Recebido em 12/07/2017. Aprovado em 09/08/2018. Avaliado pelo sistema dableblind perreview Publicado conforme normas da ABNT. http://dx.doi.org/10.22279/navus.2019.v9n1.p182-191.812

\title{
Transferência de tecnologia na política de offset: o caso do plano de expansão da radioterapia
}

\author{
Myller Augusto Santos Gomes Mestre em Políticas Públicas. Universidade Estadual do Centro-Oeste (UNIOESTE) - Brasil. \\ myller33@hotmail.com \\ João Luiz Kovaleski Doutor em Instrumentação Industrial. Universidade Tecnológica Federal do Paraná (UTFPR) - \\ Brasil. kovaleski@utfpr.edu.br \\ Regina Negri Pagani Doutora em Engenharia de Produção. Universidade Tecnológica Federal do Paraná (UTFPR) - \\ Brasil. reginapagani@utfpr.edu.br \\ Gilberto Zammar Doutor em Engenharia de Produção. Universidade Tecnológica Federal do Paraná (UTFPR) - \\ Brasil.zammar@utfpr.edu.br
}

\section{RESUMO}

Esta pesquisa objetivou identificar e compreender o processo de transferência de tecnologia promovida pela política de offset no âmbito da saúde pública, chamado Plano de Expansão da Radioterapia do Ministério da Saúde. 0 objetivo da referida política é criar e melhorar organizações credenciadas para tratamentos oncológicos a partir da inserção de equipamento radioterápico. Neste sentido, utilizou-se a estratégia metodológica do estudo de caso, amparada pela pesquisa aplicada, com abordagem qualitativa e exploratória. Analisaram-se a partir da pesquisa documental e da observação assistemática participante de que forma ocorrem a transferência de tecnologia envolvendo relações interorganizacionais para a aquisição e recebimento de equipamento radioterápico. Constatou-se que as iniciativas de inserção de equipamento radioterápico do contexto do Plano de Expansão da Radioterapia têm sofrido inúmeros enfrentamentos inseridos nas particularidades contextuais e organizacionais que afetam seu desenvolvimento e efetividade. Há desafios que exigem respostas de um conjunto de organizações envolvidas para efetivar o acordo comercial estabelecido pela política de offset, e o primeiro deles, é enaltecer a primeira etapa como processo de transferência de tecnologia.

Palavras-chave: Transferência de tecnologia. Políticas públicas. Políticas de offset.

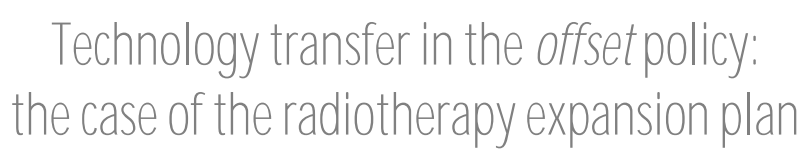

\begin{abstract}
This research aimed to identify and understand the process of technology transfer promoted by offset policy in the field of public health, called the Plan for Expansion of Radiotherapy of the Ministry of Health. The objective of this policy is to create and improve organizations accredited for cancer treatments through the insertion of radiotherapy equipment. We used the methodological strategy of the case study, supported by applied research, with a qualitative and exploratory approach. Based on documentary research and participant non-systematic observation, we analyzed how the transfer of technology involving interorganizational relations for the acquisition and reception of radiotherapy equipment takes place. We verified that the initiatives of insertion of radiotherapeutic equipment in the context of the Plan of Expansion of Radiotherapy have undergone numerous confrontations inserted in the contextual and organizational particularities that affect its development and effectiveness. There are challenges that require responses from a set of organizations involved in order to implement the trade agreement established by the offset policy, and the first one is to highlight the first stage as a process of technology transfer.
\end{abstract}

Keywords: Technology transfer. Public policy. Offset policy. 


\section{INTRODUÇÃO}

Emergentes das políticas públicas, os programas governamentais representam iniciativas do Estado em segmentos definidos enquanto áreas estratégicas como segurança, saúde, educação, economia e agricultura com a finalidade de assegurar direitos à cidadania através do acesso a serviços públicos.

As legislações de compras públicas representam uma dinamização da ação governamental, colocando regras para que a prática seja efetiva. Em um contexto informal, o surgimento do offset ou compensação comercial, industrial ou tecnológica no contexto brasileiro aconteceu nas forças armadas, onde se buscavam equipamentos tecnologicamente avançados no exterior. Em contrapartida, forneciam-se commodities e outros meios como pagamento e a exigência de transferir a tecnologia.

Partindo do que a política de offset representa a iniciativa dos processos irruptivos de transferência de tecnologia entre contextos populacionais diferentes e reconhecendo que nestes contextos existem a necessidade de compreender o fenômeno, formula-se a pergunta de pesquisa:

Como ocorrem os processos de transferência de tecnologia de equipamentos adquiridos pelo Plano de Expansão da Radioterapia?

\section{TRANSFERÊNCIA DE TECNOLOGIA}

A transferência de tecnologia (TT) é um conceito simples, mas, a partir de uma visão abrangente de todos os aspectos de uma tecnologia, sua complexidade se desenvolve (GIBSON; SMILOR, 1991; GREEN, 1999). A TT representa a movimentação da tecnologia através de algum tipo de canal: pessoa a pessoa, de grupo para grupo ou de organização para organização, sendo fundamentalmente representado pelo processo de aplicação de conhecimentos (GIBSON; SMILOR, 1991).

Nas relações interorganizacionais, Aitken e Harrison (1999) descobriram, em países que receberam investimento estrangeiro direto (IED) e, onde geralmente essa transferência ocorre de países desenvolvidos para países em desenvolvimento, a TT surge na forma de externalidade. No entanto, além de acessar essa tecnologia, os países em desenvolvimento e os ativos produtivos possibilitam no processo de $\pi$ os transbordos que podem ser utilizados e otimizados como recursos tecnológicos, know how, práticas de marketing e habilidades de gestão.

Com o foco nas relações interorganizacionais, Bozeman (2000) descreve a importância da aquisição do conhecimento tecnológico através de parceiros externos, sendo que a transferência de tecnologia envolve o know-how, conhecimento tecnológico, ou tecnologia de uma organização para outra. 0 processo de TT envolve mover uma inovação tecnológica ou conhecimento de uma organização de pesquisa e desenvolvimento até o processo de comercialização, realizado por empresas privadas, organizações de pesquisa e desenvolvimento, representadas por universidade, unidade corporativa ou por um laboratório governamental (ROGERS; TAKEGAMI; YIN, 2001).

Svedin e Stage (2016) descobriram a existência de custos de transação envolvidos no processo de TT, entretanto, a busca por ganhos necessita ser ampla para que a absorção dos custos seja justificada. Os recursos financeiros devem ser suficientes para assegurar a viabilidade do projeto, uma vez que recursos humanos capacitados são fundamentais para o êxito da transferência de tecnologia. Além disso, o processo envolve atividades de compras ou absorção de tecnologias nacionais ou estrangeiras consideradas de interesse para a capacitação tecnológica, contribuindo para o desenvolvimento econômico e social do país (BOZEMAN, 2000; BOZEMAN; RIMES; YOUTIE, 2015).

Para minimizar os impactos do processo de transferência de tecnologia, as diferentes partes interessadas com diferentes arranjos institucionais devem articular propósitos estratégicos devido aos diversos desafios impostos pelo próprio processo. Desta forma, diferenças culturais devem ser superadas para que os benefícios sejam claramente observados pelo usuário final (WISNER, 1984; THEODORAKOPOULOS; PRECIADO; BENNET, 2012). A contribuição ao desenvolvimento de capacidades inovadoras inseridas em processos de desenvolvimento de novos produtos e serviços pode advir da $\Pi$, desde que atenda o nível de desenvolvimento tecnológico que o recebedor deseja (MALM, FREDRIKSSON, J OHANSEN, 2016). 


\section{POLÍTICA DE OFFSET: A POLÍTICA DE COMPENSAÇÃO INDUSTRIAL, COMERCIAL E TECNOLÓGICA}

Considerado um fenômeno global caracterizado pela reciprocidade que envolve interações entre organizações de diferentes países, a prática de comércio compensado tem se apresentado como uma ferramenta para operações de importações comerciais, industriais e tecnológicas exigindo das organizações envolvidas um comportamento empresarial internacional (TIEN, YANG, 2005). Benefícios latentes estão inseridos nas práticas de countertrade além dos fatores motivadores que podem potencializar o crescimento social e econômico (NASSIMBENI; SARTOR, M; ORZES, 2014) acerca dos fatores motivadores, a razão maior do surgimento da prática em questão está na representatividade de uma resposta à capacidade de contratação de empréstimos dos países em desenvolvimento (ERRIDGE, ZHABYKENOV, 1998).

Na compreensão de Malm, Fredriksson e J ohansen (2016), a política de offset negocia um acordo em que o comprador inclui dentro do contrato a condição de que o vendedor tem que realizar certas atividades que beneficiam o comprador, e suas modalidades são adotadas de acordo com os critérios adequados. Uma característica relevante da prática de offset está inserida nos setores específicos da indústria, onde os preços de venda unitária são expressivos e para os compradores o papel regulatório do Estado sobre os processos de negócios internacionais são intensos em aspectos burocráticos e de valorização da indústria nacional (MACPHERSON, 2003; DORANOVA; COSTA; DUYSTERS, 2010).

O setor aeroespacial é pioneiro em offset, o início das atividades ocorreu em 1944, em Bretton Woods: com o surgimento do Banco Mundial e do Fundo Monetário Internacional, países aliados procuravam alternativas em buscar fontes de recursos financeiros e mecanismos para reestabelecimento da ordem mundial no perío do pós-guerra (TIEN; YANG, 2005; DORANOVA; COSTA; DUYSTERS, 2010). Os mecanismos de compensação, em especial o offset, apresentaram vantagens na perspectiva teórica: redução dos custos de conformidade, extensão do sinal de preço fora do limite máximo da capacidade de pagamento e dispara o processo de transferência de tecnologia (TROTIGNON, 2012).

0 offset representou para algumas nações o mecanismo ideal para sair de armadilhas econômicas, onde o desenvolvimento econômico e industrial acentuado começa a promover problemas de escassez de matéria-prima, momento em que os países em vias de desenvolvimento, considerados aliados, passaram a participar da prática compensatória, a exemplo do Plano Marshall que transferiu 13 bilhões em ajuda dos Estados Unidos à Europa Ocidental, sendo que nos anos de 1951 a 1973, excedentes agrícolas foram trocados com países aliados (DE LONG; EICHENGREEN, 1991).

Na pesquisa de Macpherson (2003), fora da indústria aeronáutica e de defesa, pouco se sabe sobre outros setores que se utilizam da prática de offset, máquinas e equipamentos que, neste contexto, representam bens de capital básicos que todas as nações devem explorar em seu processo de desenvolvimento. Essas práticas não são acompanhadas de perto pelo governo, contudo, o risco à indústria nacional, descoberta de fatores de competitividade e a prontidão de defesa podem ser afetados significativamente.

Inserida nas modalidades de comércio internacional, o offset normalmente envolve formas de partilha da produção muitas vezes representadas pelo processo de subcontratação (MACPHERSON, 2003; MULLER, 2007). Com tipologias identificadas como compensação direta que representa o processo de partilha da produção, transferência de tecnologia, treinamento de trabalhadores e a compensação indireta incluem outras formas de comércio compensatório, como por exemplo, a prática de permuta (MACPHERSON, 2003).

No Quadro 1, são apresentadas as características do processo de deslocamento, compensações diretas e indiretas. 
Quadro 1 - Características da compensação direta e indireta

\begin{tabular}{|l|l|l|}
\hline Definição & \multicolumn{1}{|c|}{ Compensação direta } & \multicolumn{1}{c|}{ Compensação Indireta } \\
\hline Classificações & $\begin{array}{l}\text { Transações diretamente relacionadas a } \\
\text { bens e serviços importados pela empresa. }\end{array}$ & $\begin{array}{l}\text { Arranjos contratuais que envolvem } \\
\text { bens e serviços não relacionados } \\
\text { diretamente com a empresa } \\
\text { contratada. }\end{array}$ \\
\hline $\begin{array}{l}\text { Ce tecnologiana, treinamento, produção, } \\
\text { produção licenciada ou atividades de } \\
\text { financiamento. }\end{array}$ & $\begin{array}{l}\text { Compras, investimentos, treinamento, } \\
\text { atividades de financiamento, } \\
\text { assistência de marketing / exportação } \\
\text { e transferência de tecnologia. }\end{array}$ \\
\hline
\end{tabular}

Fonte: Adaptado de Jang e Ryu (2008)

Com sua semelhança ao investimento estrangeiro direto (IED), a política de offset pode aumentar o crescimento econômico, tecnológico e industrial. A Organização Mundial do Comércio (OMC) destaca que difusões positivas ocorrem quando o IED é realizado, exemplos são $\Pi$ e know-how, desenvolvimento empresarial e reestruturação, integração à comunidade internacional, especialmente, no que tange ao comércio, apoio à formação de capital humano, desenvolve a competitividade da indústria nacional. Todos esses transbordamentos não parecem relevantes quando a negociação comercial é estabelecida (MENCINGER, 2003). 0 desafio da prática de offset está na internalização dos novos bens, serviços, conhecimentos, técnicas que a população selecionada irá receber.

\subsection{Política de Offsetno Brasil: Mecanismos de Regulamentação}

No Brasil, a política de offset se desenvolveu inicialmente no segmento da Força Área Brasileira (FAB) na década de 50. A aquisição de aeronaves desenvolvidas na Inglaterra representou sua primeira experiência no comércio compensatório, a contrapartida brasileira foi quantidades de algodão e a exigência da TT (IVO, 2004).

A primeira ação governamental modificando foi o Decreto no 86.010, de 15 de maio de 1981 que inseriu a cláusula de compensação com produtos nacionais (BRASIL, 1981). Com as experiências brasileiras na prática de offset, as ações realizadas no âmbito da área civil são limitadas, entretanto, o Estado com as experiências da FAB e do Exército Brasileiro em políticas de offset, levou o Ministério da Defesa a aprovar, em 2002, sua política e diretrizes de compensação comercial, industrial e tecnológica por intermédio da Portaria Normativa no 764/MD, de 27 de dezembro de 2002 que determinava como objetivos, a promoção do nível tecnológico e qualitativo da indústria de defesa, fomento e fortalecimento de setores de interesse das forças armadas, ampliação do mercado de trabalho, obtenção de recursos externos, sejam diretos ou indiretos, e incremento da nacionalização e progressão da independência do mercado (BRASIL, 2002).

Posteriormente, a Lei no 12.349 de 15 de dezembro de 2010 alterou completamente o contexto da política de offset no contexto brasileiro, compras públicas regulamentadas pela Lei $n-8.666$ de 21 de junho de 1993 e a Lei 10.973 de 02 de dezembro de 2004 sobre incentivos à inovação e à pesquisa científica e tecnológica no ambiente produtivo, sofreram alterações. (BRASIL, 2010).

A alteração significativa no âmbito das compras públicas foi nos editais de licitação para bens, serviços ou obras mediante a autorização, onde há possibilidade de exigir que o contratado promova em favor do órgão as medidas de compensação comercial, industrial e tecnológica ou acesso a condições vantajosas de financiamento (BRASIL, 2010). Ainda no âmbito das compras públicas as alterações e a inserção da prática de offset em formato de política também contemplam a modalidade pregão, regulamentada pela Lei № 10.520 de 17 de julho de 2002 (BRASIL, 2002).

A conduta da administração pública sobre a política de offset, o Decreto no 7.546, de 02 de agosto de 2011 apresenta alterações à Lei de compras públicas federais e define a medida comercial, industrial e tecnológica sendo "a prática compensatória estabelecida como condição para o fortalecimento da produção de bens, do desenvolvimento tecnológico ou da prestação de serviços, com a intenção de gerar benefícios de natureza industrial, tecnológica ou comercial concretizados em diferentes tipologias" (BRASIL, 2011). 
As tipologias do Decreto nำ 7.564, de 02 de agosto de 2011 são:

- Coprodução;

- Produção sob licença;

- Produção subcontratada;

- Investimento financeiro em capacitação industrial e tecnológica;

- Transferência de tecnologia;

- Obtenção de materiais e meios auxiliares de instrução;

- Treinamento de recursos humanos;

- Contrapartida comercial;

- Contrapartida industrial.

A Comissão Interministerial de Compras Públicas (Cl-CP) que acompanha os processos de offsets e suas proposições normativas é composta por ministros de Estado presidido pelo Ministro da Fazenda com participação dos Ministros do Planejamento, Orçamento e Gestão, do Desenvolvimento, Indústria e Comércio Exterior, da Ciência Tecnologia e Inovação e das Relações Exteriores (BRASIL, 2011). Para a Cl-CP entre as suas competências institucionais em seu artigo 8 do Decreto 7.546, cabe à Cl-CP avaliar o impacto econômico, para examinar a política de margem de preferência e de medidas de compensação nas compras públicas sobre o desenvolvimento nacional em áreas setoriais.

Assim, com a regulamentação da política de offset no contexto brasileiro permitiu a realização da compensação tecnológica entre o Ministério da Saúde e a Empresa Varian Medical Systems que segue os dispositivos legais vigentes no âmbito da administração pública federal.

\section{METODOLOGIA}

Com uma primeira experiência na utilização da política de offset com máquinas e equipamentos não oriundos do segmento de defesa, o plano de expansão da radioterapia no Sistema Único de Saúde tem como objetivo aumentar a cobertura do tratamento de doenças oncológicas através da radioterapia.

Em virtude da nova dimensão das compras públicas através da Lei no 12.349/2010, o Ministério da Saúde realizou um acordo comercial para a aquisição de oitenta (80) aceleradores lineares produzidos pela empresa americana Varian Medical Systems (BRASIL, 2016). Com preço unitário atrativo e com contrapartidas a serem realizadas pelo vendedor, denominadas de acordo de compensação tecnológica, representado pelas iniciativas de construção da fábrica para produção de aceleradores lineares no Brasil, capacitar fornecedores locais, recursos humanos e realizar a transferência de tecnologia sobre o processo de desenvolvimento de software embarcado para cinco instituições científicas e tecnológicas brasileiras.

A pesquisa bibliográfica foi realizada junto as bases de dados, Sciencedirect, Scopus e Webofscience, sem limite temporal, associando a busca pelas palavras-chaves propostas nesta investigação: technology transfer, public policy e offset policy, os achados foram selecionados pelo número de citações e contemplados no referencial proposto com intuito de servir de base de discussão com dados empíricos encontrados.

A pesquisa documental foi realizada junto ao site do Ministério da Saúde, acervo de documentos da organização contemplada, ambientes institucionais onde foram publicadas todas as informações oficiais divulgadas sobre o plano de expansão da radioterapia.

Nesta pesquisa, utilizou-se a observação assistemática participante, onde o próprio pesquisador procede de forma direta à coleta de informações, interagindo com os participantes envolvidos no objeto de pesquisa, entretanto, o delineamento da observação direta está a partir do delineamento teórico constituído (QUIVY; CAMPEMNHOUDT, 2005).

A observação assistemática participante ocorreu na organização contemplada pelo plano de expansão da radioterapia situada no Estado do Paraná, no período de junho de 2017 a outubro de 2017. 0 pesquisador participou do processo de primeiro funcionamento do equipamento acelerador linear e acompanhou, de forma remota, o processo de seleção da radioterapia externa para tratamento oncológico. Participando do fluxo de atendimentos diários, sejam consultas ou nas aplicações radioterápicas, o pesquisador percebeu a adequação da organização em questão no gerenciamento da capacidade instalada, 
antes da inauguração oficial do acelerador linear, sendo que a organização em questão já o utilizava para compor uma abrangência maior aos pacientes advindos do Sistema Único de Saúde (SUS).

Ocorreram aproximações dentro dos setores-chaves para o processo de gerenciamento do equipamento, como o setor de engenharia, setor de radioterapia, setor de gestão de pessoas e com a coordenação geral. $O$ intuito formulado foi entender como $T$ ocorreu na organização selecionada no Estado do Paraná, quais os ganhos estabelecidos em termos de transferência de tecnologia.

A análise dos dados coletados ocorreu de forma qualitativa, o que se observou foi a forma como 0 processo de transferência de tecnologia se desenvolveu, procurou ser fiel a proposta de realização proposta, e a observação na situação contemplada permitiu conhecer as qualificações e limitadores que transbordam nesta etapa significativa do plano de expansão da radioterapia.

\section{RESULTADOS E DISCUSSÕES}

Trata-se da aquisição de aceleradores lineares, um equipamento utilizado na modalidade terapêutica radioterápica externa que permite controlar a intensidade e direcionalidade dos raios ionizantes. Esse equipamento é considerado a maior referência em tratamentos radioterápicos, contudo, o determinante para a utilização deste equipamento está na localização anatômica das células malignas (BRESSAN, 2010).

Os componentes do acelerador linear são divididos em dois módulos: o primeiro com comportamento estático e o outro com comportamento móvel. Na parte estática, os componentes são o sistema de refrigeração, componentes elétricos capazes de gerar micro-ondas, na parte móvel a seção aceleradora, cabeçote e os colimadores (SOUZA, 2017).

A relação dos componentes é descrita e apresentada na Figura 1.

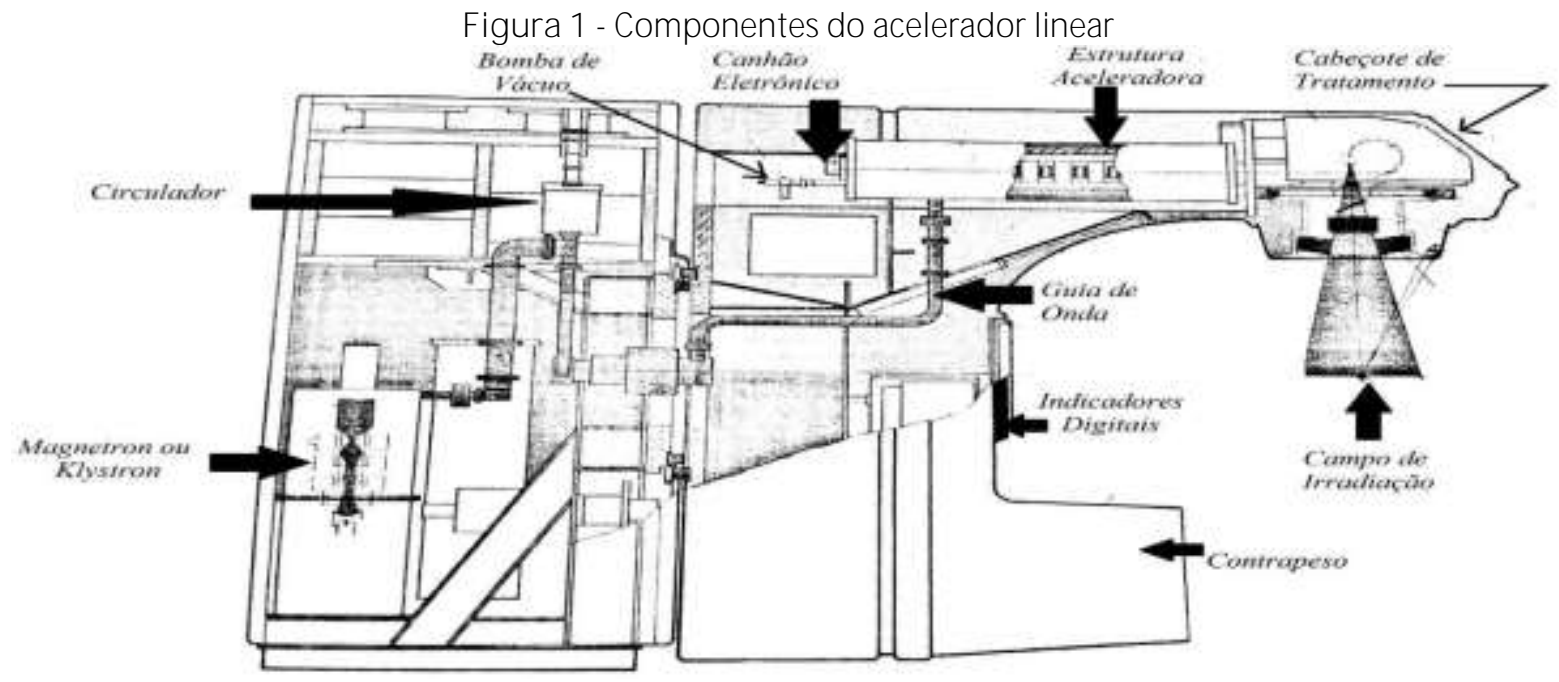

Fonte: Adaptado de Souza (2017)

O acordo comercial é representado por um processo inserido nas duas etapas descritas anteriormente que precisam ser cumpridas pelas organizações envolvidas para efetivamente inserir o acelerador linear no contexto hospitalar. A Figura 2 apresenta o fluxo de etapas que se apresenta de forma sequencial estabelecendo atos institucionais, contudo, a etapa somente é considerada concluída pelo comitê gestor onde são alinhados requisitos e adaptações necessárias a cada organização contemplada. 
Figura 2 - Fluxo das etapas do processo de inserção do acelerador linear no contexto hospitalar
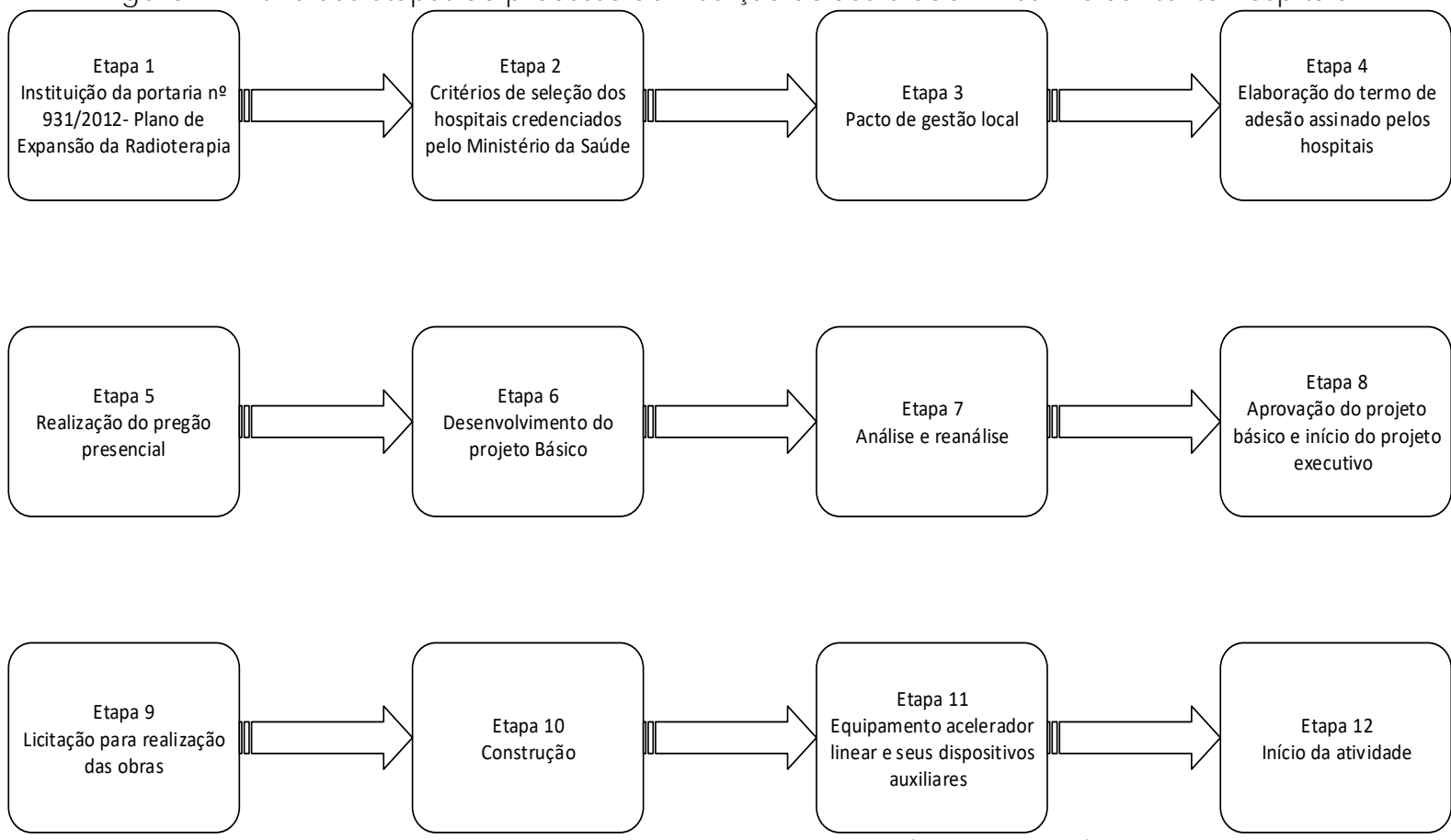

Fonte: Adaptado do Ministério da Saúde (BRASIL, 2016)

O sincronismo entre as etapas e atividades são fundamentais para a efetividade do plano, visto que há a participação de diferentes instituições como Ministério da Saúde, empresa Varian Medical Systems, Secretarias Estaduais de Saúde, os hospitais contemplados pelo plano, as construtoras responsáveis pela obra planejada. Diante disso, os esforços institucionais são relevantes para que a solução em radioterapia seja disponibilizada, mas atualmente o cenário de evolução do plano é vagaroso.

Identificar a $\Pi$ no processo de inserção do acelerador linear, dentro do fluxo de etapas, conforme figura 2, é compreendido que no desenvolvimento do projeto básico, e após a infraestrutura do banker construída, a chegada do acelerador linear e seus dispositivos auxiliares representam o processo de $\Pi$. Estas etapas representam as relações interorganizacionais estabelecidas pela política de offset, ou seja, a organização recebedora, organização detentora da tecnologia embarcada nos equipamentos e as organizações mediadoras que estabelecem a relação através da prática de comércio. Essa afirmação é sustentada pelos autores (BOZEMAN, 2000; OCKWELL et al., 2010).

Em cada etapa, os dificultadores inseridos na primeira fase, aparecem na consecução dos projetos básicos e executivos por parte das organizações contempladas. Somente a aquisição dos equipamentos e seus auxiliares representa um processo simples, entretanto, desenvolver infraestrutura para o equipamento e estabelecer sintonias entre as organizações envolvidas, depende de vários fatores como a competência e habilidades da organização construtora, os espaços da estrutura receptora, além de elementos contextuais como mão de obra, fornecedores, transporte e estruturas viárias que permitem a mobilidade de recursos necessários ao empreendimento.

A não consideração da primeira fase do plano de expansão da radioterapia como processo de $\Pi T$ ofusca a percepção de existência e contribuição. Neste contexto, é necessária uma reflexão so bre a riqueza de informações e subsídios advindos de contextos divergentes inseridos no plano, o que permite descobrir potencialidades que podem ser inseridas nos aceleradores lineares otimizando o processo de tratamento radioterápico (MULLER, 2007, NASSINBENI; SARTOR; ORZES, 2014)

$\mathrm{Na}$ situação contemplada analisada, a experiência em tratamentos oncológicos demonstrou a capacidade organizacional em absorver melhorias, mesmo havendo problemas considerados estruturais. 0 que o processo de $\Pi$ demonstra, é que não basta ter a tecnologia movida do ponto $A$ ao ponto $B$, é preciso considerar os transbordos positivos e negativos que surgem na situação em que a transferência acontece. Transbordos positivos na situação contemplada analisada são expertise com equipamentos radioterápicos, 
familiaridade com os equipamentos acelerador linear, competência operacional acerca dos profissionais envolvidos com os aceleradores linear, capacidade e habilidade em desenvolver projetos estabelecidos pelo Sistema Único de Saúde (SUS).

Sobre os transbordos negativos, condições estruturais podem representar atrasos no cronograma do projeto proposto, ausência de expertise com equipamentos aceleradores lineares, falta de capacidades e habilidades organizacionais em gerenciar os projetos providos pelo SUS, habilidades consideradas relacionais entre empresas, onde a relação interorganizacional pode ser comprometida impactando no processo de $\Pi$, fragilizando a iniciativa governamental de inserção tecnológica voltada a ampliação do tratamento oncológico realizado pela saúde pública.

\section{CONSIDERAÇÕES FINAIS}

Após o mapeamento dos fluxos de seleção de hospitais, bem como as etapas a serem cumpridas, verificou-se que dentro deste processo existem etapas que são propensas ao contexto da $\Pi$, visto que a sua materialização depende exclusivamente das relações interorganizacionais. É preciso estabelecer que a inserção do acelerador linear corresponde ao processo de TT, e seu comportamento não é similar, pois em cada contexto em que o processo de inserção procura se desenvolver, as particularidades do contexto externo e interno realizaram interferências que comprometeram os objetivos estabelecidos pela política de offset. As dificuldades enfrentadas pela iniciativa governamental não somente acontecem no ponto alto do plano que seria a inserção do acelerador linear nos hospitais, mas na adequação das estruturas para recebimento de melhorias ou novas instalações.

Sobre os processos de $T$ inseridos na política de offset, inicialmente parecem em segundo plano, pois a aquisição e a relação comercial se tornam o foco principal, contudo, o transbordamento efetivo e positivo da $\Pi$ pode contribuir para o desenvolvimento tecnológico das organizações recebedoras, alcançar o crescimento econômico e intensificar as relações internacionais com foco em comercialização, $\Pi$, pesquisa e desenvolvimento de produtos e formação de know-how, e o transbordamento negativo é a não realização da $\Pi$, o upgrade dos equipamentos não acontece e a otimização e a efetivação do acordo comercial se torna limitada. Assim, o ideal é realizar a $\Pi T$ primeiramente, antes da chegada dos equipamentos. Desta forma, 0 melhoramento pode ocorrer antecipadamente e não observar a TT somente como contrapartida ao acordo comercial.

O estudo sobre a política de offset voltada à saúde pública revelou a complexidade das atividades desenvolvidas com dependência das relações interorganizacionais, onde atividades estruturadas e o processo decisório centralizado acabam tornando os procedimentos vagarosos e instáveis. A iniciativa é válida, contudo, se considerar o processo de inserção de equipamentos como $T$ e reflexionar sobre os contextos ambientais com vistas às adequações, o que pode representar ganhos estratégicos e operacionais para as instituições envolvidas.

\section{REFERÊNCIAS}

AITKEN, Brian. J.; HARRISON, Ann. E. Do domestic firms benefit from direct foreign investment? Evidence from Venezuela. American Economic Review, v. 89, n. 3, p. 605-618, 1999.

BLOHMKE, Julian. Technology complexity, technology transfer mechanisms and sustainable development. Energy for Sustainable Development, v. 23, p. 237-246, 2014.

BOZEMAN, Barry. Technology transfer and public policy: a review of research and theory. Research Policy, v. 29, n. 4, p. 627-655, 2000.

BOZEMAN, Barry.; RIMES, Heather.; YOUTIE, Jan. The evolving state-of-the-art in technology transfer research: Revisiting the contingent effectiveness model. Research Policy, v. 44, n. 1, p. 34-49, 2015. 
BRASIL. Decreto no 86.010, de 15 de maio de 1981. Dispõe sobre a Comissão de Coordenação do Transporte Aéreo Civil - COTAC do Ministério da Aeronáutica. Diário Oficial da União, Brasília, DF, seção 1, p. 8926-8927, 18 maio 1981.

BRASIL. Ministério da Defesa. Portaria Normativa no 764/MD. Aprova a Política e Diretrizes de Compensação Comercial, Industrial e Tecnológica do Ministério da Defesa. Diário Oficial da União, Brasília, DF, seção 1, p. 19, 31 dez. 2002.

BRASIL. Lei no 12.349, de 15 de dezembro de 2010. Altera as Leis n 8.666, de 21 de junho de 1993, 8.958, de 20 de dezembro de 1994. Diário Oficial da União, Brasília, DF, seção 1, p. 2, 16 dezembro 2010.

BRASIL. Decreto no 7.546, de 02 de agosto de 2011. Regulamenta o disposto nos $\S \S 50$ a 12 do art. 30 da Lei no 8.666, de 21 de junho de 1993, e institui a Comissão Interministerial de Compras Públicas. Disponível em:< http://www.planalto.gov.br/ccivil_03/_Ato2011-2014/2011/Decreto/D7546.htm>. Acesso em: 21 fev. 2017.

BRASIL. Ministério da Saúde. Plano de expansão da radioterapia. 2012. Disponível em: <http://portalms.saude.gov.br/ciencia-e-tecnologia-e-complexo-industrial/complexo-industrial/plano-deexpansao-da-radioterapia-no-sus>. Acesso em: 16 nov. 2016.

BRESSAN, Aparecida Isabel. A disponibilidade de aceleradores lineares para o tratamento do câncer no Brasil e as teses de focalização e simplificação do SUS. 2010. 71 p. Dissertação (Mestrado em Ciências da Saúde Pública) - Fundação Oswaldo Cruz. Rio de Janeiro, 2010.

DE LONG, Bradford.; EICHENGREEN, Barry. The marshall plan: history's most successful structural adjustment program. Cambridge: National Bureau of Economic Research, 1991.

DORANOVA, Asel; COSTA, lonara; DUYSTERS, Geert. Knowledge base determinants of technology sourcing in clean development mechanism projects. Energy Policy, v. 38, n. 10, p. 5550-5559, 2010.

ERRIDGE, Andrew; ZHABYKENOV, Dubek. The role of purchasing in countertrade. European Journal of Purchasing \& Supply Management, v. 4, n. 2, p. 97-107, 1998.

GIBSON, David V.; SMILOR, Raymond W. Key variables in technology transfer: a field-study based empirical analysis. Journal of Engineering and Technology Management, v. 8, n. 3, p. 287-312, 1991.

GREEN, David. Cross cultural technology transfer of sustainable energy systems: a critical analysis. Renewable Energy, v. 16, n. 1, p. 1133-1137, 1999.

IVO, Ronan Coura. A prática do OFFSET como instrumento dinamizador do desenvolvimento industrial e tecnológico. 2004. 157 p. Dissertação (Mestrado em Desenvolvimento Sustentável) - Universidade de Brasília, Brasília, 2004.

JANG, Won-J oon.; RYU, J in Young. Technology valuation model in defense offset trade: income approach. In: PORTLAND INTERNATIONAL CONFERENCE ON MANAGEMENT OF ENGINEERING \&TECHNOLOGY, 2008, Portland. Proceedings. Portland: Portland State University. p. 2521-2534, 2008.

MACPHERSON, Alan. The impact of industrial offsets on the US machine tool industry. Competition \& Change, v. 7, n. 2-3, p. 101-112, 2003.

MALM, Anna. Margaretha; FREDRIKSSON, Anna.; J OHANSEN, Kerstin. Bridging capability gaps in technology transfers within related offsets. J ournal of Manufacturing Technology Management, v. 27, n. 5, p. 640-661, 2016.

MENCINGER, J oze. Does foreign direct investment always enhance economic growth? Kyklos, v. 56, n. 4, p. 491-508, 2003. 
MULLER, Adrian. How to make the clean development mechanism sustainable: the potential of rent extraction. Energy Policy, v. 35, n. 6, p. 3203-3212, 2007.

NASSIMBENI, Guido; SARTOR, Marco; ORZES, Guido. Countertrade: compensatory requests to sell abroad. Journal for Global Business Advancement, v. 7, n. 1, p. 69-87, 2014.

OCKWELL, David G et al. Intellectual property rights and low carbon technology transfer: conflicting discourses of diffusion and development. Global Environmental Change, v. 20, n. 4, p. 729-738, 2010.

QUIVY, Raymond; CAMPENHOUDT, Luc Van. Manual de investigação em ciências sociais. 4. ed. Lisboa: Gradiva, 2005.

ROGERS, Everett M.; TAKEGAMI, Shiro.; YIN, Jing. Lessons learned about technology transfer. Technovation, v. 21, n. 4, p. 253-261, 2001.

SOUZA, Anderson Sorgatti de. Estudo comparativo dos parâmetros associados à dose absorvida e controle de qualidade em aceleradores lineares com filtro aplainador e sem filtro aplainador. 2017. 66 p. Dissertação (Mestrado em Ciências) - Instituto de Pesquisas Energéticas e Nucleares, São Paulo, 2017.

SVEDIN, Dick; STAGE, J esper. Impacts of foreign direct investment on efficiency in Swedish manufacturing. Springer Plus, v. 5, n. 1, p. 614, 2016.

TIEN, Mo-Chung.;YANG, Ching-chow. Taiwan's ICP mechanism: a review and a stage approach. Technological Forecasting and Social Change, v. 72, n. 1, p. 29-48, 2005.

TROTIGNON, Raphael. Combining cap-and-trade with offsets: lessons from the EU-ETS. Climate Policy, v. 12, n. 3, p. 273-287, 2012.

THEODORAKOPOULOS, Nicholas.; PRECIADO, Deycy J aneth Sanchez.; BENNETT, David. Transferring technology from university to rural industry within a developing economy context: the case for nurturing communities of practice. Technovation, v. 32, n. 9, p. 550-559, 2012.

WISNER, Alain. Vers une anthropotechnologie. Paris: CNAM, 1984 